\title{
Liquid Level Sensor Utilising a Long Period Fiber Grating
}

\author{
S. Grice*a, W. Zhang ${ }^{\mathrm{a}}$, K Sugden ${ }^{\mathrm{a}}$, I. Bennion ${ }^{\mathrm{a}}$ \\ ${ }^{a}$ Photonics Research Group, Aston University, Birmingham, B4 7ET, United Kingdom
}

\begin{abstract}
We propose here a liquid level sensor using a long period fiber grating (LPFG) in which direct liquid level measurement is carried out by utilising an LPFG, $100 \mathrm{~mm}$ in length and a periodicity of $1 \mathrm{~mm}$. The LPFG was exposed to liquids with varying levels and the wavelength shift of a selected loss band of the transmission spectra was monitored using a broadband light source and an optical spectrum analyzer. The mechanism of this LPFG sensor is based on the fact that the effective Refractive Index (RI) of a cladding mode is directly dependant on the RI of the surrounding medium, be it air, or in this case water and petrol. As the surrounding RI changes, so does the phase matching condition of the LPFG. The result for the level change of the liquid with a specific RI is both a shift in wavelength and a change in the attenuation level of the selected loss band. For the selected loss band, continuous wavelength shifts of $9.5 \mathrm{~nm}$ and $25 \mathrm{~nm}$ for $100 \mathrm{~mm}$ of water and petrol level change have been observed respectively, with sub-millimetre accuracy.
\end{abstract}

Keywords: Liquid Level Sensing, Long Period Fiber Grating (LPFG), Refractive Index (RI), Broad Band Source (BBS), Optical Spectrum Analyzer (OSA).

\section{INTRODUCTION}

Long Period Fiber Gratings (LPFGs) are in-fiber optical devices which couple forward prorogating light from the fiber core mode into the co-propagating fiber cladding modes of Single Mode Fiber (SMF), and typically range between $100 \mu \mathrm{m}$ up to many $\mathrm{mm}$ in length. They are extensively used in both sensing and communications applications. LPFGs have a number of characteristics that are advantageous over other sensors including lightness and compactness, low insertion loss and relative ease of fabrication.

A great deal of interest has been focused on executing liquid-level measurements in numerous areas, such as the in the fuel, oil, and water tanks of aircraft, rockets, and automobiles. In many cases, electrical sensors are inappropriate or simply not feasible because of the hazards posed by sparks in potentially explosive environments. Electrical sensors will also be undesirable when electromagnetic interference can overwhelm the signals generated by them. Liquid level sensors using optical fibers hold particular promise as replacements for many existing sensing technologies, especially in harsh environments. Sensors based on purely optical techniques have been previously described [1-2]. Optical fiber Bragg grating (FBG) techniques attract much attention due to their significant feature of wavelength encoding, which allows the removal of influences from system variables such as source intensity and attenuation. Most of those reported FBG sensors based on the measurements of strain, pressure and weight are greatly affected by environmental variables such as temperature, pressure and gravity. A proposed technique in which a LPFG was used for direct level measurement has been previously reported [3], however, it is based on measuring the attenuation of the loss band of the transmission spectra, which still suffers the influence of system variables. In this work we propose a direct liquid level measurement technique by utilizing an LPFG to monitor the wavelength shift of a selected attenuation band. Attenuation band wavelength shifts of $9.5 \mathrm{~nm}$ and $25 \mathrm{~nm}$ for $100 \mathrm{~mm}$ of water and petrol level change have been observed in the experiments, respectively.

*gricesj@aston.ac.uk; phone 44121204 3545; fax 44121204 8382; www.ee.aston.ac.uk/research/prg

Optical Components and Materials VI, edited by Shibin Jiang, Michel J. F. Digonnet,

John W. Glesener, J. Christopher Dries, Proc. of SPIE Vol. 7212, 72120C

(C) 2009 SPIE · CCC code: 0277-786X/09/\$18 - doi: 10.1117/12.809104

Proc. of SPIE Vol. 7212 72120C-1 


\section{THEORY}

\subsection{LPFG Refractive Index Sensitivity}

LPFGs can be manufactured either by mechanical induction, or by permanently photo-inducing a modulation of the RI of the fiber core of Single Mode Fiber (SMF), which is achieved by exposing the fiber to ultraviolet (UV) irradiation. The latter manufacturing technique can either be inscribing on a point-by-point basis, or by exposing the entire length of fiber simultaneously by means of an amplitude mask. By observing the transmission spectrum, distinct attenuation loss bands are evident at discrete wavelengths. These loss bands are created because the fundamental core mode is coupled to the forward propagating cladding modes.

The resonant wavelength, $\lambda_{\text {res, }}$ is defined as follows [4].

$$
\lambda_{\text {res }}=\left(n_{c o}^{e f f}-n_{c l, m}^{e f f}\right) \Lambda
$$

Where $\Lambda$ is the grating period and $n_{c o}^{e f f}$ and $n_{c l, m}^{e f f}$ are the effective indexes of the fundamental core mode and the $m^{\text {th }}$ cladding mode, respectively. $n_{c o}^{e f f}$ can be regarded as independent of the surrounding RI, $n_{s u r}$, however $n_{c l, m}^{e f f}$ can not. This is due to the light in the core being bound within the core, thus not interacting with the surrounding medium. Therefore only the cladding modes that extend out of the fiber are affected by changes to the surrounding medium RI. Using equation (1), the expression for the surrounding RI sensitivity can be derived [5].

$$
\frac{d \lambda_{\text {res }}}{d n_{\text {sur }}}=\lambda_{\text {res }} \cdot \gamma \cdot\left(\Gamma_{\text {sur }}\right)
$$

where $\Gamma_{\text {sur }}$ represents the RI dependence, and $\gamma$ is the waveguide dispersion derived as [6].

$$
\gamma=\frac{\frac{d \lambda_{r e s}}{d \Lambda}}{\left(n_{c o}^{e f f}-n_{c l, m}^{e f f}\right)}
$$

and the RI dependence, $\Gamma_{\text {sur }}$, is derived as

$$
\Gamma_{s u r}=-\frac{u_{m}^{2} \lambda_{r e s}^{3} n_{s u r}}{8 \pi r_{c l}^{3} n_{c l}\left(n_{c o}^{e f f}-n_{c l, m}^{e f f}\right)\left(n_{c l}^{2}-n_{s u r}^{2}\right)^{3 / 2}}
$$

where $u_{m}$ is the $m$ th root of the zero order Bessel function of the first kind, $n_{c l}$ and $n_{c o}$ are the elasto-optic coefficients of the cladding and core. Whilst the above equation (4) is relatively complicated, a good approximation of $\Gamma_{\text {sur }}$ is,

$$
\Gamma_{\text {sur }} \propto \frac{1}{\left(n_{c o}^{e f f}-n_{c l, m}^{e f f}\right)\left(n_{c l}^{2}-n_{s u r}^{2}\right)^{3 / 2}}
$$




\section{EXPERIMENT}

\subsection{LPFG Fabrication}

The LPFG used for this experiment was manufactured using a frequency doubled CW argon laser. The fiber, Corning SMF-28, was exposed to UV irradiation of $244 \mathrm{~nm}$ using the point-by-point inscription technique. The UV fabrication configuration is illustrated in Figure 1.

The principal of operation is that the incident laser beam is focused onto the optical fiber by employing cylindrical focusing lenses, which focuses the light in the $\mathrm{x}$ direction. The system also comprises of a controllable translation stage, which translates from left to right along the fiber, enabling the pre-programmed shutter-controlled laser light to inscribe the LPFG by the point-by-point technique. The parameters that can be pre-programmed into the system are the grating period, $\Lambda$, and the speed of the translation stage. These are programmed depending on the LPFG specifications.

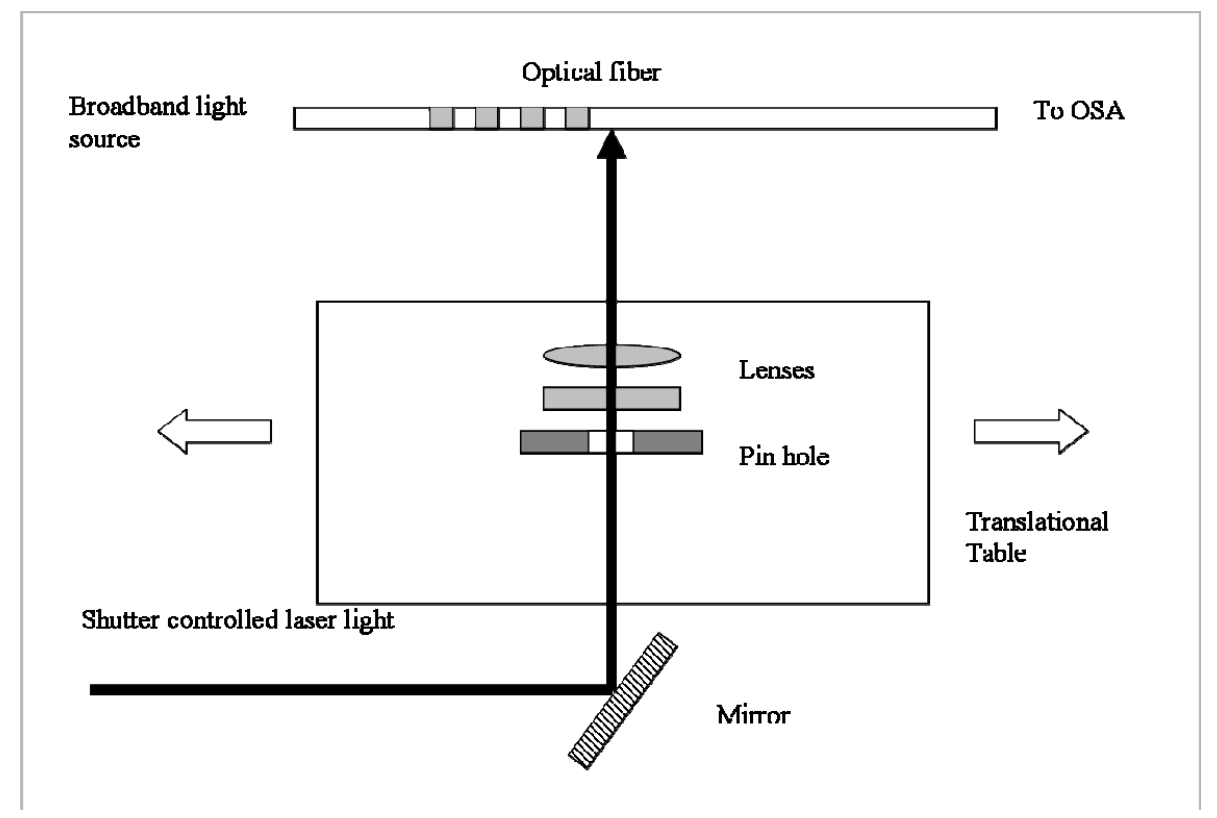

Fig. 1. Laser system configuration for writing Long Period Fiber Gratings using the point-by-point inscription technique.

As previously stated, for the investigations performed, the fiber used was Corning SMF-28, which was first hydrogen loaded before exposing it to the UV light. The hydrogen in the core acts to increase the photosensitivity of the fiber, any unused hydrogen will diffuse out over a period of time after the exposure. Once the LPFG was written, the fiber was placed in an oven for 24 hours at $80^{\circ} \mathrm{C}$ for annealing, this acts to stabilise the grating spectrum. The grating written for these experiments was $100 \mathrm{~mm}$ in length and had a periodicity of $1000 \mu \mathrm{m}$.

\subsection{Experimental set-up}

The basic configuration for the liquid level sensor concept is depicted in Figure 2.

The system consists of a Broad Band Source (BBS) and an Optical Spectrum Analyzer (OSA). Light from the source propagates along the optical fiber, and upon reaching the LPFG, light is coupled from the core mode to the cladding modes. As the OSA only interrogates the light in the core, wavelengths coupled to the cladding appear missing from the transmission spectrum, known as attenuation or loss bands.

The system is based on measuring the wavelength shift of a selected loss band of the transmission spectra of the LPFG. The LPFG was exposed to a variety of liquids of varying RI and the wavelength of the selected loss band was monitored using the BBS and OSA with the resolution set to $0.1 \mathrm{~nm}$. In this work the LPFG was suspended between two fixed points, and placed with-in a glass tube with a tap at the bottom to control liquid level. The glass tube was marked with a 
ruler to indicate the liquid level. With the LPFG fixed in a constant position, as the liquid level changes, so does the amount of the LPFG that is covered by said liquid.

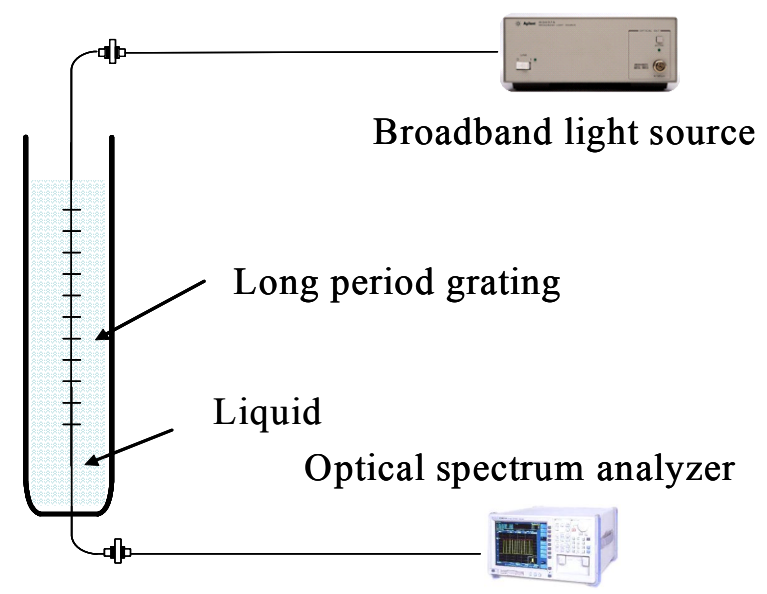

Fig. 2. Experimental configuration for the Long Period Fiber Grating based liquid level sensor.

\subsection{Measurement results}

Figure 3 shows the measured transmission spectra of the LPFG when it was fully immersed in air, water and fuel (unleaded petrol), respectively.

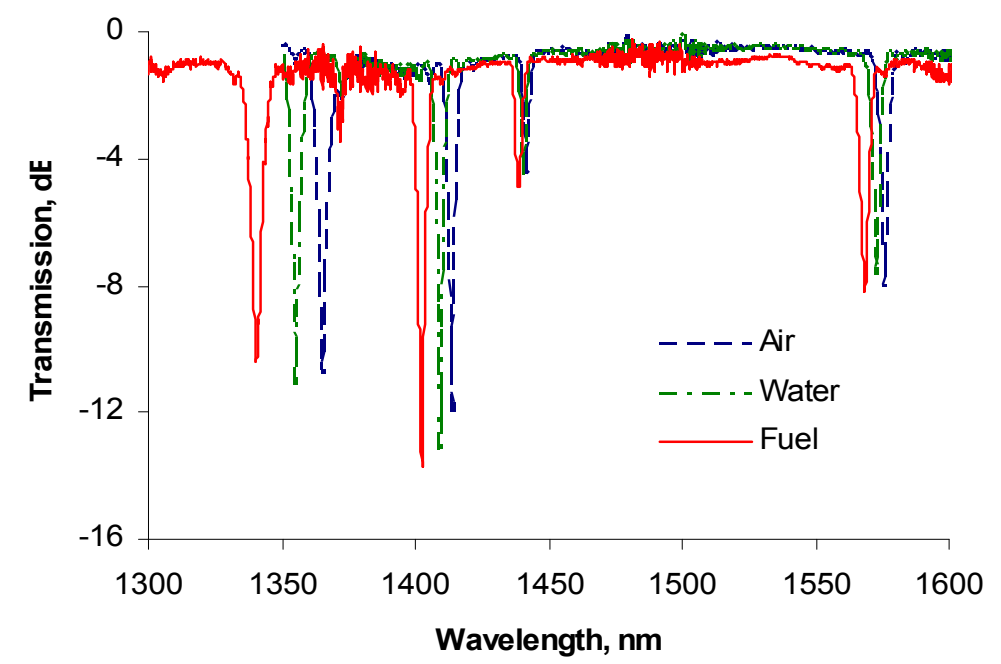

Fig. 3. Transmission spectra of the Long Period Fiber Grating in air, water and fuel.

If one considers the loss band around $1360 \mathrm{~nm}$, which shows the highest sensitivity in this case, the shift of this resonant wavelength is $9.5 \mathrm{~nm}$ over a $100 \mathrm{~mm}$ water level range and $25 \mathrm{~nm}$ over a $100 \mathrm{~mm}$ fuel level range. The detailed changes of resonant wavelength against water level change is depicted in figure $4 a$, from which one can clearly see the resonant wavelength of the loss band exhibits a blue shift against the increased water level. Note that $5 \mathrm{~dB}$ of transmission loss offset has been added to each measurement to increase the clarity of the result. The measured spectra without offset in transmission are shown in figure $4 \mathrm{~b}$. One can notice that there are two small loss bands aside the main loss band, whose wavelengths, marked as $\lambda_{1}$ and $\lambda_{2}$, do not vary with the changing water level. This interesting feature could be very useful in real applications as these two loss bands can be used to calibrate the sensor output against any possible cross sensitivity. 


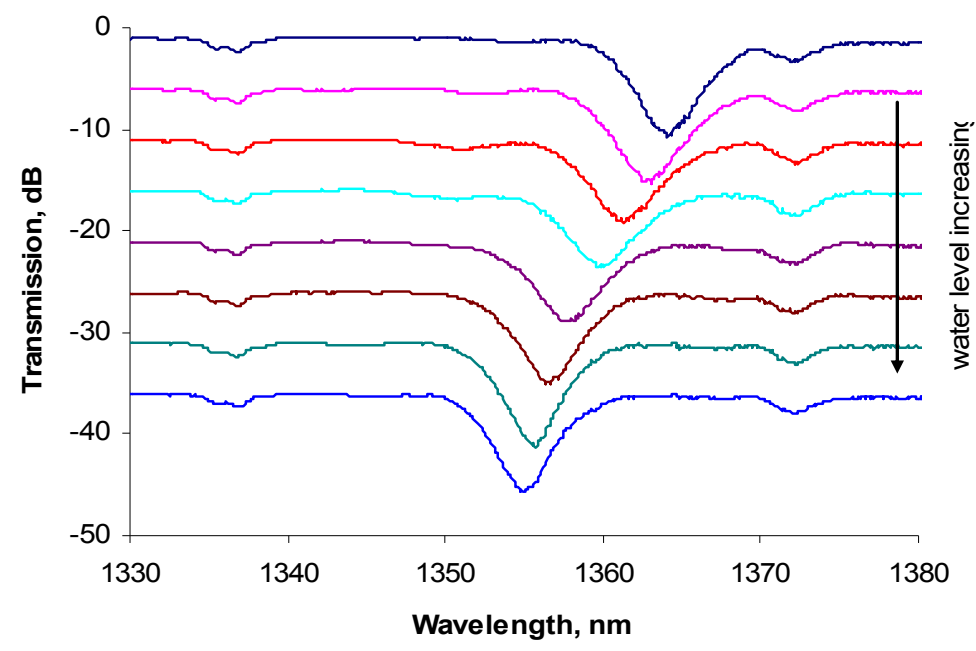

Fig. 4a. Resonant peak shift with increasing water level with $5 \mathrm{~dB}$ transmission loss offsets added for clarity.

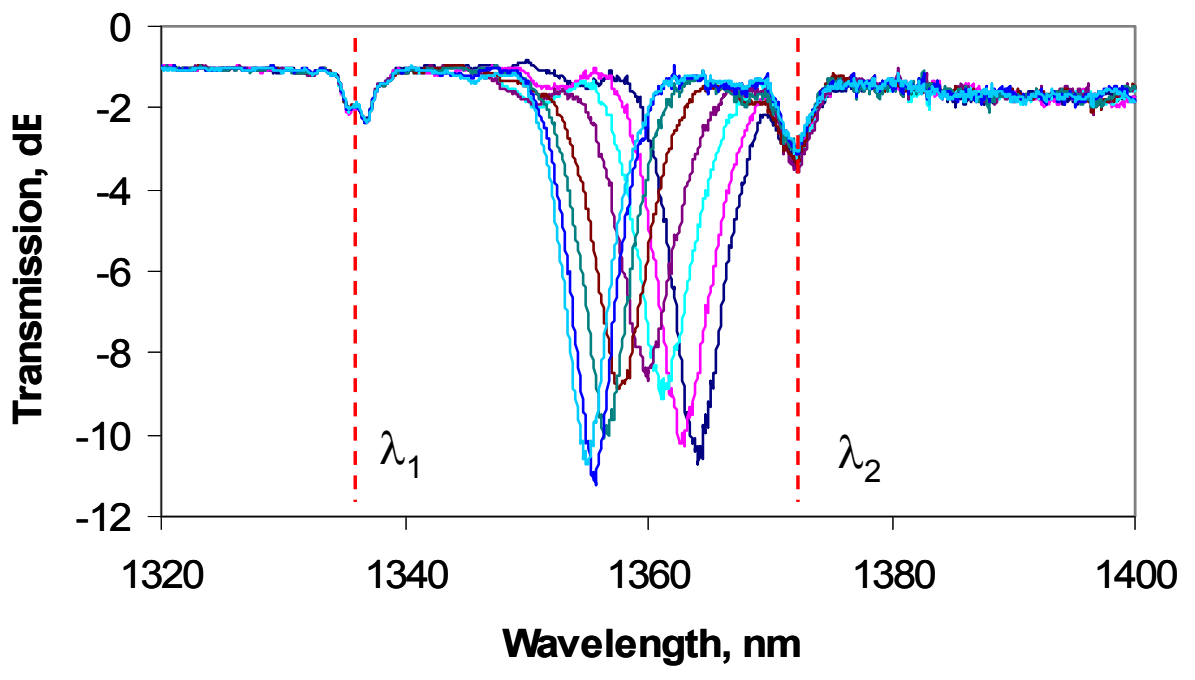

Fig. 4b. Resonant peak shift with increasing water level without $5 \mathrm{~dB}$ transmission loss.

Figure 5 illustrates a full water cycle measurement. As can be seen, there is little hysteresis within the interested $10 \mathrm{~cm}$ range of the sensor output. The difference between the outputs of increased and decreased water levels mainly arose from the reading error of the water level. The sensor output shows some aberrancy when the water level was below the LPFG. This is attributed to the tension induced by the liquid. Once the LPFG is immersed in water, this tension does not affect the sensor performance. 


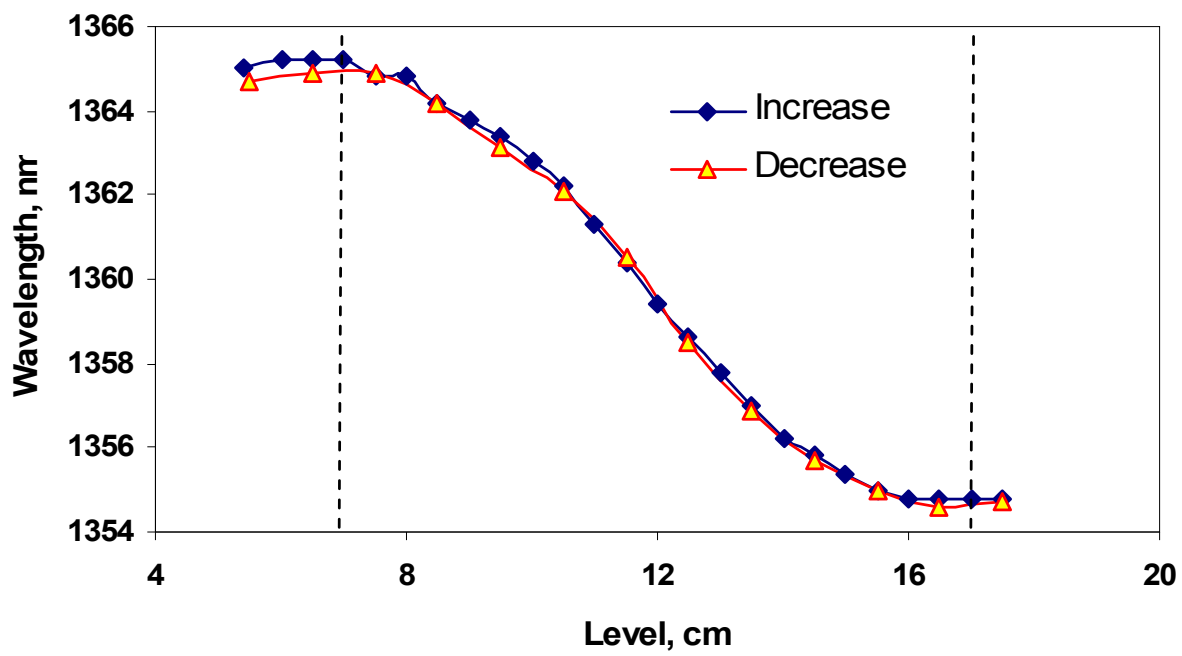

Fig. 5. Water level measurement cycle.

The measurements on fuel level were also carried out using the same experimental arrangement. The wavelength shift of the same transmission loss band was measured against the varying fuel level. The captured spectra are shown in 3D view in figure 6. As we mentioned above the selected transmission loss band shows a $25 \mathrm{~nm}$ wavelength shift over $10 \mathrm{~cm}$ fuel level change. However, from the consecutively captured spectra one can notice that the selected loss band had a blue shift and disappeared when the fuel level increased; then a new loss band generated at shorter wavelength (around 1340 $\mathrm{nm}$ ) and still had a blue shift, but at a slower rate, as the fuel level increased. This is not really desired as this means that one could lose the measurement information for a certain range. We therefore turned to another transmission loss band around $1414 \mathrm{~nm}$, which is less sensitive to the fuel level change than that at $\sim 1360 \mathrm{~nm}$.

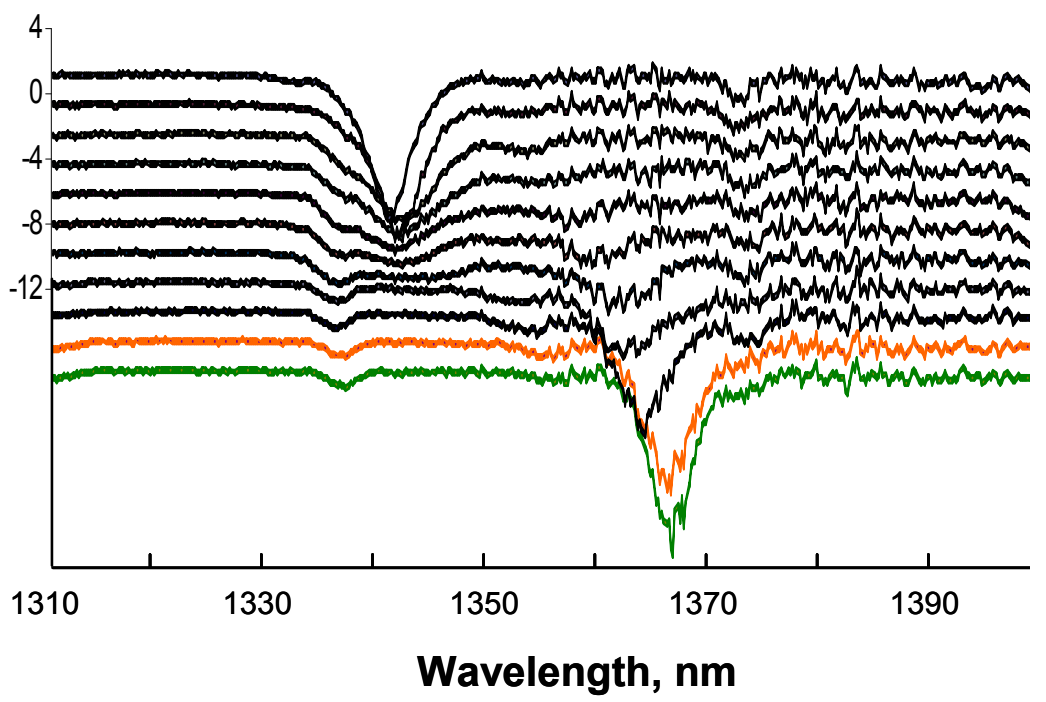

Fig. 6. 3D spectra illustrating wavelength shift induced by exposure to petrol.

The consecutively captured spectra against varying petrol level are shown in the figure 7 with $5 \mathrm{~dB}$ transmission offset for each measurement. From it one can clearly see that the wavelength of the selected loss band around $1414 \mathrm{~nm}$ continuously shifts toward the shorter wavelength while the fuel level was increasing. It also shows a good sensitivity of $11 \mathrm{~nm}$ of the wavelength shift over $10 \mathrm{~cm}$ fuel level change. 


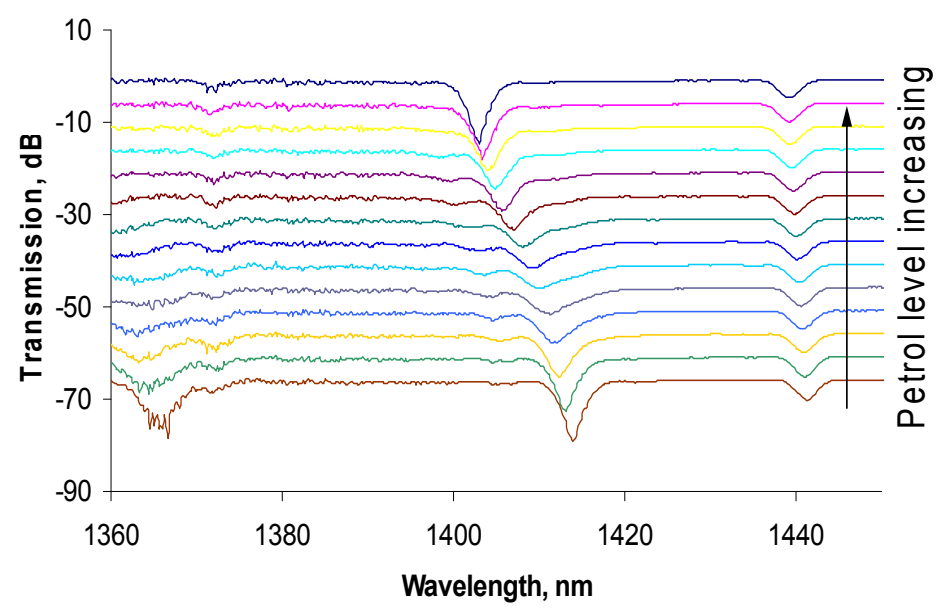

Fig. 7. Spectra illustrating wavelength shift with $5 \mathrm{~dB}$ offset added for clarity.

\section{DISCUSSION \& CONCLUSIONS}

In this paper we have demonstrated the capability of using an LPFG as a liquid level sensor by means of measuring the wavelength shifts of a particular attenuation band, induced by external RI variations. We have shown that for $100 \mathrm{~mm}$ of water and fuel level change, wavelength shifts of $10 \mathrm{~nm}$ and $25 \mathrm{~nm}$ have been induced on the $1360 \mathrm{~nm}$ attenuation band, as well as an $11 \mathrm{~nm}$ shift in fuel for the $1414 \mathrm{~nm}$ attenuation band.

The sensitivity of LPFGs arises from the fact that the effective refractive index of a cladding mode is dependent on the RI of the surrounding medium. When the external RI changes, the phase matching condition of the grating changes, resulting in the changes in both wavelength and attenuation of the LPFG loss band [7]. The wavelengths of the transmission bands of the LFPG normally show a negative shift as long as the RI of the surrounding medium is lower than that of the cladding. The closer to the RI of the cladding gives larger negative wavelength shift [8]. This explains why $10 \mathrm{~cm}$ fuel level induced larger wavelength shift than $10 \mathrm{~cm}$ water level in this work.

Generally the surrounding medium of an LPFG is air with the refractive index of 1 . If the LPFG is partly immersed in the liquid to be measured, the result is two LPFGs are formed in series. The second (new) LPFG has the length equal to the immersed length and the surrounding RI equal to that of the liquid. If the dispersion condition of the LPFG is properly chosen by choosing the appropriate grating period, the combined spectral response of this pair of LPFGs exhibits a shift in the resonant wavelength of selected loss band at the rate associated with the immersed length. When the LPFG is fully immersed in the liquid the spectral response is that of the LPFG with the external RI of the liquid. Therefore by monitoring the wavelength change of the selected loss band, one can measure the immersed length of the grating, thus the liquid level.

\section{AKNOWLEDGMENTS}

The work described is part of the Integrated Wing collaborative R\&T programme and is funded by the UK Technology Strategy Board. We wish to acknowledge the helpful discussions we have had with our partners in this project.

\section{REFERENCES}

[1] D. A. Jackson, "High precision remote liquid level measurement using a combination of optical radar and optical fibers," in Proc. First Int. IEE Con. Optical Fiber Sensors (London, England), 1983, vol. 221, pp. 100-103.

[2] T. Guo, etc., "Temperature-insensitive fiber Bragg grating liquid-level sensor based on bending cantilever beam", IEEE Photon. Technol. Lett., Vol. 17, 2005, pp. 2400-2402. 
[3] S. Khaliq, S. W. James, and R. P. Tatam, "Fiber-optic liquid-level sensor using a long-period grating", Optics Lett., 26, 2001, pp. 1224-1226.

[4] A.M. Vengsarker, P.J. Lemaire, J.B. Judkins, V. Bhatia, T. Erdogan, J.E. Sipe. "Long Period Fiber Gratings As Band-Rejection Filters" Journal of lightwave technology, Vol. 14, pp. 58-64, Jan. 1996.

[5] X. Shu, T. Allsop, B. Gwandu, L. Zhang, and I. Bennion, "Room-tem-perature operation of widely tunable loss filter," Electron. Lett., vol. 37, pp. 216-218, Feb. 2001.

[6] T. W. MacDougall, S. Pilevar, C. W. Haggans, and M. A. Jackson, "Generalized expression for the growth of long period gratings,” IEEE, Photon. Technol. Lett., vol. 10, pp. 1449-1451, Oct. 1998.

[7] H.J. Patrick, A Kersey and F Bucholtz, "Analysis of the Response of Long Period Fiber Gratings to External Index of Refraction", Journal Of Lightwave Technology, Vol. 16, no. 9, September 1998, pp. 1606-1612.

[8] N. D. Rees, S. W. James, R. P. Tatam, and G. J. Ashwell, "Optical fiber long-period gratings with Langmuir-Blodgett thin-film overlays", Optics Lett., 27, 2002, pp. 686-688 